\title{
Aetiological diagnosis of acute respiratory failure in critical care oncology
}

\author{
Anne-Pascale Meert and Jean-Paul Sculier
} Affiliations: Université Libre de Bruxelles (ULB), Institut Jules Bordet, Service des Soins Intensifs et Urgences
Oncologiques, Brussels, Belgium.

Correspondence: A-P. Meert, Institut Jules Bordet, Rue Héger-Bordet, 1, B-1000 Bruxelles, Belgium. E-mail: ap.meertabordet.be

First steps for a new noninvasive approach for the aetiological diagnosis of ARF in cancer patients http://ow.ly/kPvZj

Acute complications are frequent in cancer patients and their adequate management, including in critical care units, may be crucial [1]. The prognosis of such complications in terms of intensive care unit (ICU) and hospital mortality is mainly determined by the acute physiological perturbations they induce, as reflected by gravity scores in critically ill cancer patients $[2,3]$, but also by the degree of life-supporting therapy that is required, such as mechanical ventilation $[3,4,5]$ or extra renal epuration [6]. This has been confirmed by many other studies summarised in a systematic review of the literature [7]. After recovery from the acute complication, the further prognosis is determined by the characteristics of the underlying cancer and no longer related to the perturbations induced by the complication [1].

The most frequent cause of ICU admission in cancer patients is acute respiratory failure (ARF). It can be due to a direct manifestation of the malignant disease (for example by airway obstruction or pleural effusion), treatment toxicity (for example by radiation or drug pneumonitis) or indirect complications, such as infections or haemorrhages.

Improved critical care management with the use of noninvasive ventilation or invasive protective mechanical ventilation has allowed achievement of better results over the past two decades, particularly in the critically ill haematological patient. Nevertheless, ARF requiring intubation and mechanical ventilation is still associated with $50-70 \%$ mortality [8-11]. Failure of an initial noninvasive ventilation trial, requiring intubation and invasive support, is associated with a poor prognosis [12-13].

Another important aspect of in the management of such cancer patients is the treatment of the underlying cause of the ARF. In order to see a rapid improvement of ARF, the cause should be treatable and rapidly reversible. For instance, it has been shown that documented or clinically suspected bacterial infections in severely ill patients with haematological malignancies have a better outcome than other complications [14]. These data support the idea that improving diagnostic strategies might be important.

In the present issue of the European Respiratory Journal, a strategy, called DIRECT, is proposed on the basis of six categories of criteria for identifying the cause of ARF [15]. DIRECT is a mnemonic for: Delay since malignancy onset or haematopoietic stem-cell transplantation since symptom onset and since antibiotics/ prophylaxis implementation; pattern of Immune deficiency; Radiographic appearance; Experience and knowledge of the literature; and Clinical picture findings by high-resolution computed Tomography of the chest. The six criteria have been defined based on the clinical experience of the authors. The purpose of DIRECT is to obtain, at the time of ICU admission, a rapid noninvasive aetiological diagnosis in ARF in order to allow prompt initiation of the causal treatment by avoiding potentially dangerous and/or timeconsuming invasive approaches, such as bronchoalveolar lavage (BAL). 
To assess the usefulness of this approach, the authors investigated, in a retrospective series of cancer patients from their hospital, the association between the DIRECT categories and three diagnostic groups of ARF (bacterial pneumonias, opportunistic pulmonary infections and non-infectious lung disorders). The study included 424 patients with documented diagnoses: 201 bacterial pneumonia, 131 opportunistic infections, and 92 non-infectious disorders. Bacterial pneumonia was shown to be associated with neutropenia, solid tumour, multiple myeloma, time since symptom onset $<3$ days, shock, unilateral crackles and unilateral chest radiograph pattern. Opportunistic infections were associated with steroids, lymphoproliferative disorder and stem cell transplant. Non-infectious disorders were associated with acute leukaemia. The categories were strongly associated with the causes of ARF in cancer patients. In the future, DIRECT might be used for developing an algorithm for selecting first-line diagnostic investigations and empirical treatments, in order to reduce the need for potentially harmful examinations, to decrease unnecessary processing time and to diminish costs.

There are some limitations in this study. The most important limitation was not including 230 patients because of unknown, suspected or miscellaneous cause of ARF, which represented about one-third of the overall population. The article does not provide a lot of information about this group of patients. They will, nevertheless, represent a significant part of the patients to be included in the future prospective study. Moreover, the study includes a very large number of haematological malignancies. Solid tumours represent only $15 \%$ of the cases, while they are much more frequent than haematological malignancies. This peculiar selection of patients makes extrapolation of the results to a general cancer population difficult. More data on this category of patients would be useful for designing the prospective study. The category entitled "Experience and knowledge of the literature" (the E of DIRECT) is very soft and has not been assessed in the reported study. In addition, for the category T of DIRECT, high-resolution chest tomography has been performed in only $49 \%$ of the cases.

One question deriving from the DIRECT study is the place of the bronchoalveolar lavage. The team from Saint-Louis Teaching Hospital, Paris, France has already shown that, in their experience, BAL performed in the ICU, did not significantly decrease intubation requirements in critically ill cancer patients with ARF [16]. Nevertheless, BAL is often performed (65\% of the cases in the present study), even before ICU admission. Moreover, with the current supportive techniques, such as noninvasive ventilation, performing BAL in the ICU is also easier and safer.

The present study assessing a new noninvasive approach for the aetiological diagnosis of ARF in cancer patients has to be considered as a first step. More data are necessary to better define the categories in the different types of cancer patients and to set up a reproducible algorithm. Finally, validation by an adequately sized prospective study is needed before application in routine practice.

\section{References}

1 Sculier JP, Paesmans M, Markiewicz E, et al. Scoring systems in cancer patients admitted for an acute complication in a medical intensive care unit. Crit Care Med 2000; 28: 2786-2792.

2 Mokart D, van Craenenbroeck T, Lambert J, et al. Prognosis of acute respiratory distress syndrome in neutropenic cancer patients. Eur Respir J 2012; 40: 169-176.

3 Adam AK, Soubani AO. Outcome and prognostic factors of lung cancer patients admitted to the medical intensive care unit. Eur Respir J 2008; 31: 47-53.

4 Vallot F, Paesmans M, Berghmans T, et al. Leucopenia is an independent predictor in cancer patients requiring invasive mechanical ventilation: a prognostic factor analysis in a series of 168 patients. Support Care Cancer 2003; 11: 236-241.

5 Meert AP, Close L, Hardy M, et al. Noninvasive ventilation: application to the cancer patient admitted in the intensive care unit. Support Care Cancer 2003; 11: 56-59.

6 Berghmans T, Meert AP, Markiewicz E, et al. Continuous venovenous haemofiltration in cancer patients with renal failure: a single-centre experience. Support Care Cancer 2004; 12: 306-311.

7 den Boer S, de Keizer NF, de Jonge E. Performance of prognostic models in critically ill cancer patients - a review. Crit Care 2005; 9: R458-R463.

8 Meert AP, Sotiriou M, Berghmans T, et al. Non-invasive ventilation in cancer patients: a historically matched controlled study. Hospital Chronicles 2006; 1: 93-98.

9 Depuydt PO, Benoit DD, Vandewoude $\mathrm{KH}$, et al. Outcome in noninvasively and invasively ventilated hematologic patients with acute respiratory failure. Chest 2004; 126: 1299-1306.

10 Depuydt PO, Benoit DD, Roosens CD, et al. The impact of the initial ventilatory strategy on survival in hematological patients with acute hypoxemic respiratory failure. J Crit Care 2010; 25: 30-36.

11 Azoulay E, Alberti C, Bornstain C, et al. Improved survival in cancer patients requiring mechanical ventilatory support: impact of noninvasive mechanical ventilatory support. Crit Care Med 2001; 29: 519-525.

12 Meert AP, Berghmans T, Markiewicz E, et al. Invasive mechanical ventilation in cancer patients. Prior non invasive ventilation is a poor prognostic factor. J BUON 2011; 16: 160-165.

13 Gristina GR, Antonelli M, Conti G, et al. Noninvasive versus invasive ventilation for acute respiratory failure in patients with hematologic malignancies: a 5-year multicenter observational survey. Crit Care Med 2011; 39: 2232-2239. 
14 Benoit DD, Depuydt PO, Peleman RA, et al. Documented and clinically suspected bacterial infection precipitating intensive care unit admission in patients with hematological malignancies: impact on outcome. Intensive Care Med 2005; 31: 934-942.

15 Schnell D, Mayaux J, Lambert J, et al. Clinical assessment for identifying causes of acute respiratory failure in cancer patients. Eur Respir J 2013; 42: 435-443.

16 Azoulay E, Mokart D, Lambert J, et al. Diagnostic strategy for hematology and oncology patients with acute respiratory failure: randomized controlled trial. Am J Respir Crit Care Med 2010; 182: 1038-1046. 